Pacific Journal of Mathematics

RANDOM FIXED POINT THEOREMS FOR MULTIVALUEI 


\title{
RANDOM FIXED POINT THEOREMS FOR MULTIVALUED MAPPINGS
}

\author{
HEINZ W. ENGL
}

\begin{abstract}
We give some random fixed point theorems for random operators which are defined on subsets of a separable Banach space and whose values are subsets of the Banach space. The domains are allowed to be random. One of the results is a stochastic version of the Bohnenblust-Karlin-Kakutani fixed point theorem for set-valued maps.
\end{abstract}

1. Introduction. The Prague school of probabilists in the Fifties introduced the study of random fixed point theorems (cf. e.g., [10]). Recently the interest in these questions has been revived, especially by the review article [3]. Answers to some of the research problems mentioned there have been given in [5], [6], [7]. In this paper we will answer the research problem asking for a stochastic version of the Bohnenblust-Karlin fixed point theorem for set-valued maps ([4], cf. also [18]), which was proved for finite dimensional spaces by Kakutani.

A random fixed point theorem for another class of set-valued maps was recently proved in [13]. A good historic survey about fixed point theorems for set-valued maps can be found in [9].

2. Definitions and preliminary results. Throughout this paper, let $X$ be a real separable Banach space, $(\Omega, \mathscr{A}, \mu)$ a $\sigma$-finite measure space. We will use the words "stochastic" and "random" interchangeably also if $\mu$ is not a probability measure. By $2^{X}$ we denote $\{A / A \subseteq X \wedge A \neq \phi \wedge A$ closed $\}$, by $C B(X)=\left\{A / A \in 2^{X} \wedge A\right.$ bounded $\}$ and by $C C(X)=\left\{A / A \in 2^{X} \wedge A\right.$ convex $\}$.

Definition 1. Let $C: \Omega \rightarrow 2^{X}$ be a set-valued map. We call $C$ "measurable" iff for all open $D \leqq X,\{\omega \in \Omega / C(\omega) \cap D \neq \phi\} \in \mathscr{A}$. (Note that this is called "weakly measurable" in [12].) We call $C$ "separable" iff it is measurable and there exists a countable set $Z \subseteq X$ such that for all $\omega \in \Omega, \operatorname{cl}(Z \cap C(\omega))=C(\omega)$. The "graph of $C$ " is defined as $\operatorname{Gr} C=\{(\omega, x) \in \Omega \times X / x \in C(\omega)\}$.

It can be easily shown that if $C$ is measurable and has closed, convex, and solid (i.e., nonempty interior) values, then $C$ is separable. The definition of separability implies that $C$ has closed values.

Definition 2. Let $C \subseteq X$ be closed. $T: C \rightarrow 2^{X}$ is called "upper semicontinuous (usc)" iff for all $x \in C, T(x)$ is compact and for all 
closed $A \subseteq X,\{x \in C / T(x) \cap A \neq \phi\}$ is closed. $T$ is called "compact" iff it is usc and $\mathrm{cl}\left(\mathbf{U}_{x \in C} T(x)\right)$ is compact.

Unfortunately the definitions of upper semicontinuity vary in literature. We will need the following

Lemma 3. Let $C \leqq X$ be closed, $T: C \rightarrow 2^{X}$ such that $\mathrm{cl}\left(\bigcup_{x \in C} T(x)\right)$ is compact. Then the following are equivalent:

(1) $\{(x, y) \in C \times X / y \in T(x)\}$ is closed in the product-topology.

(2) For all $\left(x_{n}\right) \in C^{N}, x \in C, y_{n} \in T\left(x_{n}\right), y \in X$ such that $\left(x_{n}\right) \rightarrow x$ and $\left(y_{n}\right) \rightarrow y$ we have $y \in T(x)$.

(3) For all $x \in C, \varepsilon>0$, there exists $a \delta>0$ such that for all $z \in C$ with $\|z-x\|<\delta$ we have $\sup _{y \in T(z)} d(y, T(x))<\varepsilon$, where $d$ is the distance induced by the norm.

(4) For all $x \in C$ and all open $D \subseteq X$ with $T(x) \leqq D$ there exists a neighborhood $U$ of $x$ such that for all $z \in U \cap C, T(z) \cong D$.

(5) $T$ is compact in the sense of Definition 2.

Proof. $\quad(1) \Leftrightarrow(2)$ : obvious.

$(1) \Leftrightarrow(4): \quad[2$, p. 118].

$(4) \Leftrightarrow(5)$ : follows from [2, p. 115].

$(4) \Rightarrow(3)$ : Let $x \in C$ and $\varepsilon>0 ; D=\bigcup_{z \in T(x)}\{y \in X /\|y-z\|<\varepsilon\}$ is open and contains $T(x)$. Therefore there exists a $\delta>0$ such that for all $z \in C$ with $\|z-x\|<\delta, T(z) \subseteq D$, in other words $\sup _{y \in T(z)} d(y, T(x))<\varepsilon$.

$(3) \Rightarrow(2)$ : Let $\left(x_{n}\right) \in C^{N}$ with $\left(x_{n}\right) \rightarrow x$ and $y_{n} \in T\left(x_{n}\right)$ with $\left(y_{n}\right) \rightarrow y$ and choose $\varepsilon>0$. Then there exists a $n_{0} \in N$ such that for all $n \geqq n_{0}, d\left(y_{n}, T(x)\right)<\varepsilon \cdot 2^{-1}$ and $\left\|y_{n}-y\right\|<\varepsilon \cdot 2^{-1}$; therefore $d(y, T(x))<\varepsilon$. As $\varepsilon$ was arbitrary and $T(x)$ is closed, $y \in T(x)$.

Definition 4. Let $C$ be a mapping from $\Omega$ to $2^{X}$. A mapping $T: \operatorname{Gr} C \rightarrow 2^{X}$ is called "set-valued random operator with stochastic domain $C$ " iff $C$ is measurable and for all $x \in X$ and open $D \subseteq X$, $\{\omega \in \Omega / x \in C(\omega) \wedge T(\omega, x) \cap D \neq \phi\} \in \mathscr{A}$. Such a $T$ will be called "usc" ("compact") iff for all $\omega \in \Omega, T(\omega, \cdot)$ is usc (compact). A function $x: \Omega \rightarrow X$ will be called "random fixed point of $T$ " iff for $\mu$-almost all $\omega \in \Omega, x(\omega) \in C(\omega)$ and $x(\omega) \in T(\omega, x(\omega))$ and $x$ is measurable (i.e., for all open $D \subseteq X,\{\omega \in \Omega / x(\omega) \in D\} \in \mathscr{A})$.

Proposition 5. Let $C: \Omega \rightarrow 2^{X}$ be separable and $T: \operatorname{Gr} C \rightarrow C C(X)$ a compact set-valued random operator with stochastic domain $C$. We define $H: \operatorname{Gr} C \rightarrow C C(X)$ by

$$
H(\omega, x)=\bigcap_{n \in N} \operatorname{cl} \operatorname{conv} \underset{\substack{z \in Z \cap C \\\|z-x\|<n^{-1}}}{\bigcup} T(\omega, z),
$$

where $Z$ is a countable set as it appears in Definition 1. Then, 
(1) For all $(\omega, x) \in \operatorname{Gr} C, \phi \neq H(\omega, x) \subseteq T(\omega, x)$.

(2) For all $\omega \in \Omega$ and $x \in C(\omega) \cap Z, H(\omega, x)=T(\omega, x)$.

(3) $H$ is (product-) measurable (i.e., for all open $D \subseteq X$, $\{(\omega, x) \in \Omega \times X / x \in C(\omega) \wedge H(\omega, x) \cap D \neq \phi\} \in \mathscr{A} \times \mathscr{B}$, where $\mathscr{B}$ is the $\sigma$-algebra on $X$ generated by the open sets).

(4) For all $\omega \in \Omega, H(\omega, \cdot)$ is compact.

(5) For all (product-) measurable functions $\alpha: \Omega \times X \rightarrow X$ with $a(\omega, x) \in C(\omega)$ for all $\omega \in \Omega$ and $x \in X$ we have:

$$
\begin{aligned}
H(\cdot, a(\cdot, \cdot)): \Omega \times X & \longrightarrow C C(X) \\
(\omega, x) & \longrightarrow H(\omega, a(\omega, x))
\end{aligned}
$$

is (product-) measurable (i.e., for all open

$$
D \subseteq X,\{(\omega, x) \in \Omega \times X / H(\omega, a(\omega, x)) \cap D \neq \phi\} \in \mathscr{A} \times \mathscr{B}) .
$$

Proof. As (1), (2), (4) say something about the properties of $H$ for fixed $\omega$, we do not write the argument $\omega$ in their proofs.

(1) Let $L$ be a finite subset of $N, k=\max L, x \in C$. Because $T$ is compact, the sets cl conv $\bigcup_{\|z-x\|<n^{-1}, z \in Z \cap C} T(z)$ are closed subsets of a compact set which is independent of $n$; this is a consequence of Mazur's theorem on the closed convex hull of a compact set. Because of

$$
\phi \neq \operatorname{cl} \operatorname{conv} \underset{\substack{z \in Z \cap C \\\|z-x\|<k^{-1}}}{\bigcup} T(z) \cong \bigcap_{n \in L} \operatorname{cl} \operatorname{conv} \underset{\substack{z \in Z \cap C \\\|z-x\|<n^{-1}}}{\bigcup} T(z)
$$

the family $\left\{\right.$ cl conv $\left.\bigcup_{z \in Z \cap c, \| z-x||<n^{-1}} T(z) / n \in N\right\}$ has the finite intersection property, therefore $H(x) \neq \phi$.

Let $\varepsilon>0$ be arbitrary, but fixed and choose $n \in N$ such that for all $z \in Z \cap C$ with $\|z-x\|<n^{-1}$, $\sup _{y \in T(z)} d(y, T(x))<\varepsilon$, which is possible because of Lemma 3(3). Then for all such $z, T(z) \subseteq(T(x))_{\varepsilon}=$ $\{u \in X / d(u, T(x))<\varepsilon\}$ and because of the convexity of $(T(x))_{\varepsilon}, H(x) \subseteq$ cl conv $\bigcup_{z \in Z \cap C,\|z-x\|<n^{-1}} T(z) \subseteq \operatorname{cl}(T(x))_{\varepsilon}$. As $\varepsilon$ was arbitrary, $H(x) \subseteq$ $T(x)$.

(2) Let $x \in C \cap Z$. Then for all $n \in N, T(x) \subseteq \bigcup_{z \in C \cap z,\|z-x\|<n^{-1}} T(z)$, therefore $T(x) \subseteq H(x)$. Together with (1) we get $T(x)=H(x)$.

(4) Let $\left(x_{k}\right) \in C^{N}$ with $\left(x_{k}\right) \rightarrow x \quad(C$ is closed, therefore $x \in C)$, $y_{k} \in H\left(x_{k}\right)$ with $\left(y_{k}\right) \rightarrow y$. Let $n \in N$ be arbitrary, but fixed and choose $K \in N$ so that for all $k \geqq K,\left\|x_{k}-x\right\|<(2 n)^{-1}$. For all these $k$ we have

$$
y_{k} \in H\left(x_{k}\right) \leqq \mathrm{clconv} \underset{\substack{z \in Z \cap C \\\left\|z-x_{k}\right\|<(2 n)-1}}{\bigcup} T(z) \leqq \operatorname{clconv} \underset{\substack{z \in Z \pi n C \\\|z-x\|<n^{-1}}}{\bigcup} T(z) .
$$

Therefore $y \in \mathrm{cl}$ conv $\bigcup_{z \in Z \cap C,\|z-x\|<n^{-1}} T(z)$; because $n$ was arbitrary, this implies $y \in H(x)$. All $H(x)$ are compact, so $H$ is usc. Because of (1) and the compactness of $T$ also $H$ is compact. 
(3) Let for all $n \in N, T_{n}: \operatorname{Gr} C \rightarrow\{A \subseteq X / A \neq \phi\}$ be defined by $T_{n}(\omega, x):=\bigcup_{z \in Z \cap C(\omega),\|z-x\|<n^{-1}} T(\omega, z)$ and let $D \subseteq X$ be open. Then,

$$
\begin{aligned}
&\left\{(\omega, z) \in \operatorname{Gr} C / T_{n}(\omega, x) \cap D \neq \phi\right\} \\
&= \bigcup_{z \in Z}\left[\left\{(\omega, x) \in \operatorname{Gr} C /\|z-x\|<n^{-1}\right\} \cap(\{\omega \in \Omega / z \in C(\omega)\right. \\
&\wedge T(\omega, z) \cap D \neq \phi\} \times X)] \\
&= \bigcup_{z \in Z}\left[\left(\Omega \times\left\{x \in X /\|z-x\|<n^{-1}\right\}\right) \cap \operatorname{Gr} C\right. \\
&\cap(\{\omega \in \Omega / z \in C(\omega) \wedge T(\omega, z) \cap D \neq \phi\} \times X)] \in \mathscr{A} \times \mathscr{B},
\end{aligned}
$$

because $T$ is a set-valued random operator and $\operatorname{Gr} C \in \mathscr{A} \times \mathscr{B}$ (cf. [12, Theorem 3.3]). Theorefore $T_{n}$ is measurable as a set-valued function on the measurable space $(\operatorname{Gr} C,(\mathscr{A} \times \mathscr{B}) \cap \operatorname{Gr} C)$, and so is cl $T_{n}$ (cf. [12, Proposition 2.6]). Now we can apply [12, Theorem 9.1] and get that cl conv $T_{n}=$ conv cl $T_{n}$ is measurable (on $\operatorname{Gr} C$ ). As all cl conv $T_{n}(\omega, x)$ are compact by Mazur's theorem, we can apply [12, Theorem 4.1] and get that $H=\bigcap_{n \in N}$ cl conv $T_{n}$ is measurable as a function on $(\operatorname{Gr} C,(\mathscr{A} \times \mathscr{B}) \cap \mathrm{Gr} C)$. But this means that $\{(\omega, x) \in \Omega \times X / x \in C(\omega) \wedge H(\omega, x) \cap D \neq \phi\} \in \mathscr{A} \times \mathscr{B}$.

(5) The function $(\omega, x) \rightarrow(\omega, a(\omega, x))$ is $\mathscr{A} \times \mathscr{B}-\mathscr{A} \times \mathscr{B}-$ measurable. Using this and (3) we get that for every open $D \subseteq X$,

$$
\begin{aligned}
& \{(\omega, x) \in \Omega \times X / H(\omega, a(\omega, x)) \cap D \neq \phi\} \\
& \quad=\{(\omega, x) \in \Omega \times X /(\omega, a(\omega, x)) \in\{(\tilde{\omega}, z) \in \operatorname{Gr} C / H(\tilde{\omega}, z) \cap D \neq \dot{\phi}\}\} \in \mathscr{A} \times \mathscr{B} .
\end{aligned}
$$

Lemma 6. Let $(W, S)$ be a measurable space, $R: W \rightarrow 2^{X}$ measurable and $x: W \rightarrow X$ measurable. Then $d(x(\cdot), R(\cdot))$ is measurable as a function from $W$ into $\boldsymbol{R}$.

Proof. Let for the first part of the proof $w \in W$ be arbitrary, but fixed; therefore we do not write the argument $w$. Let $Z$ be a countable dense subset of $X$. Let $\varepsilon>0, x \in X$. If there is a $z \in Z$ with $\|x-z\|<\varepsilon \cdot 2^{-1}$ and $d(z, R)<\varepsilon \cdot 2^{-1}$, then $d(x, R)<\varepsilon$. Let now $d(x, R)<\varepsilon$. Then there exists a $r \in R$ with $\|x-r\|<\varepsilon$. Define $y=(x+r) \cdot 2^{-1}$ and $0<\delta<(\varepsilon-\|x-r\|) \cdot 2^{-1}$. Then there exists a $z \in Z$ with $\|z-y\|<\delta$. For this $z$ we have:

$$
\|x-z\| \leqq\|x-y\|+\|y-z\|=\|x-r\| \cdot 2^{-1}+\|y-z\|<\varepsilon \cdot 2^{-1}
$$

and analogously $\|z-r\|<\varepsilon \cdot 2^{-1}$, therefore $d(z, R)<\varepsilon \cdot 2^{-1}$. So we have:

$\{w \in W / d(x(w), R(w))<\varepsilon\}$

$$
=\bigcup_{z \in Z}\left[\left\{w \in W /\|x(w)-z\|<\varepsilon \cdot 2^{-1}\right\} \cap\left\{w \in W / d(z, R(w))<\varepsilon \cdot 2^{-1}\right\}\right] \in S
$$


because of the measurability of $x$ and [12, Theorem 3.3].

3. A stochastic fixed point theorem of Bohnenblust-KarlinKakutani-type.

TheOREM 7. Let $C: \Omega \rightarrow C C(X)$ be measurable with solid values. We assume that there exists a measurable $x_{0}: \Omega \rightarrow X$ with $x_{0}(\omega) \in$ int $C(\omega)$ for all $\omega \in \Omega$. Let $T$ be a compact set-valued random operator with stochastic domain $C$ such that for all $(\omega, x) \in \operatorname{Gr} C, T(\omega, x)$ is a convex subset of $C(\omega)$. Then $T$ has a random fixed point.

Proof. (a) First we assume that $(\Omega, \mathscr{A}, \mu)$ is complete. Because of the remark after Definition $1, C$ is separable. We define $H$ as in Proposition 5 and $b: \Omega \times X \rightarrow \bar{R}$ by $b(\omega, x)=\sup \left\{t \geqq 0 / x_{0}(\omega)+\right.$ $\left.t \cdot\left(x-x_{0}(\omega)\right) \in C(\omega)\right\}$. Let

$$
\begin{aligned}
a: \Omega \times X & \longrightarrow X \\
\quad(\omega, x) & \longrightarrow\left\{\begin{array}{cll}
x & \text { if } & x \in C(\omega) \\
x_{0}(w)+b(\omega, x) \cdot\left(x-x_{0}(\omega)\right) & \text { if } & x \notin C(\omega)
\end{array}\right.
\end{aligned}
$$

and $N: \Omega \times X \rightarrow \boldsymbol{R}$ be defined by $N(\omega, x)=\|x-a(\omega, x)\|+d(a(\omega, x)$, $H(\omega, a(\omega, x)))$. It is clear that $N(\omega, x)=0$ if and only if $x \in C(\omega)$ and $x \in H(\omega, x) . a$ is measurable in $\omega$ ([6, Lemma 4]) and continuous in $x$ (proof of Lemma 7 in [6]). By [12, Theorem 6.1] $\alpha$ is (product-) measurable. Furthermore for all $(\omega, x) \in \Omega \times X$ we have $a(\omega, x) \in$ $C(\omega)$. It follows from Proposition 5(5) and Lemma 6 that $N$ is measurable as a function on the measurable space $(\Omega \times X, \mathscr{A} \times \mathscr{B})$.

Now we define $F: \Omega \rightarrow\{A / A \subseteq X\}$ by

$$
F(\omega)=\{x \in X / x \in C(\omega) \wedge x \in H(\omega, x)\}=\{x \in X / N(\omega, x)=0\} .
$$

Because of Proposition 5 (parts (1) and (4)) $H(\omega, \cdot)$ fulfills the assumptions of [18, Theorem 9.2.3] on $C(\omega)$, so $F(\omega) \neq \phi$. Because of the upper semicontinuity of each $H(\omega, \cdot)$ (Proposition 5(4)) and Lemma $3(2)$ each $F(\omega)$ is closed. Because of the measurability of $N$

$$
\{(\omega, x) \in \Omega \times X / x \in F(\omega)\}=N^{-1}(\{0\}) \in \mathscr{A} \times \mathscr{B} .
$$

Now we can apply Theorem 3.5(iii) of [12] and get that $F$ is measurable (in the sense of Definition 1). Because of the KuratowskiRyll-Nardzewski-selection-theorem $([14$, p. 398]) there exists a measurable $x: \Omega \rightarrow X$ such that for all $\omega \in \Omega, x(\omega) \in F(\omega)$, which means $x(\omega) \in C(\omega)$ and $x(\omega) \in H(\omega, x(\omega)) \subseteq T(\omega, x(\omega)$ ) (Proposition 5(1)). So $x$ is a random fixed point of $T$.

(b) Let now $(\Omega, \mathscr{A}, \mu)$ be not necessarily complete; by $\left(\Omega, \mathscr{A}^{*}, \mu^{*}\right)$ we denote the Lebesgue-completion. Since $\mathscr{A} \subseteq \mathscr{A}^{*}$, the assump- 
tions of the theorem are also fulfilled with $\left(\Omega, \mathscr{A}^{*}, \mu^{*}\right)$ instead of $(\Omega, \mathscr{A}, \mu)$. Because of (a) there exists an $\mathscr{A}^{*}$-measurable $x^{*}: \Omega \rightarrow X$ with $x^{*}(\omega) \in C(\omega) \cap T\left(\omega, x^{*}(\omega)\right)$ for all $\omega \in \Omega$. Let $\mathscr{D}$ be a countable generator of $\mathscr{B}$ which exists because of the separability of $X$. Then for all $D \in \mathscr{D}, x^{*-1}(D)=A_{D} \cup L_{D}$ with $A_{D} \in \mathscr{A}, L_{D} \subseteq N_{D}, N_{D} \in \mathscr{A}$ and $\mu\left(N_{D}\right)=0$. Let $M=\bigcup_{D \in ;} N_{D}$ and define

$$
\begin{aligned}
x: \Omega & \longrightarrow X \\
& \omega \longrightarrow\left\{\begin{array}{ccc}
x^{*}(\omega) & \text { if } & \omega \notin M \\
0 & \text { if } & \omega \in M .
\end{array}\right.
\end{aligned}
$$

Then $x$ is a random fixed point of $T$.

Of course we can also get the usual corollaries like the following Rothe-type theorem:

THEOREM 8. Let $C$ and $x_{0}$ be as in Theorem 7 and $T$ be a compact set-valued random operator with stochastic domain $C$ such that for all $\omega \in \Omega$ and $x \in b d C(\omega), T(\omega, x)$ is a convex subset of $C(\omega)$. Then $T$ has a random fixed point.

Proof. Analogous to the last proof; instead of [18, Theorem 9.2.3] we use [18, Theorem 9.2.4].

REMARK 9. In the special case that all $T(\omega, x)$ are singletons (so $T$ is just an ordinary random operator) Theorem 7 is just a stochastic version of Schauder's fixed point theorem. This specialization can be done for all other fixed point theorems in this paper.

REMARK 10. The last two theorems contain the assumption that there exists a measurable $x_{0}: \Omega \rightarrow X$ such that $x_{0}(\omega) \in \operatorname{int} C(\omega)$ for all $\omega \in \Omega$ ("measurable selector of int $C$ "). This assumption has been discussed in [6]. There it turns out that in some applications it can be verified very easily (cf. the proof of [6, Theorem 20] and [6, Theorem 21]); furthermore it has been proved that if $(\Omega, \mathscr{A}, \mu)$ is complete, $C$ is uniformly bounded with closed, convex, and solid values and $X$ is reflexive then a measurable selector of int $C$ always exists ([6, Theorem 12]).

The assumption, that $C$ has to have solid values, can be done away with in the case that we consider a fixed (not randomly varying) domain of our random operator:

TheOREM 11. Let $K \subseteq X$ be nonvoid, closed and convex, $T$ : 
$\Omega \times K \rightarrow C C(X)$ a compact set-valued random operator such that $T(\Omega \times K) \subseteq K$. Then $T$ has a random fixed point.

Proof. Analogous to the proof of Theorem 7; instead of the function $N$ there we use

$$
\begin{aligned}
& N: \Omega \times K \longrightarrow \boldsymbol{R} \\
& (\omega, x) \longrightarrow d(x, H(\omega, x))
\end{aligned}
$$

which is measurable on $(\Omega \times K,(\Omega \times K) \cap(\mathscr{A} \times \mathscr{B}))$, as can be shown like in the proof of Theorem 7 .

4. Stochastic fixed point theorems for continuous set-valued random operators. The second class of set-valued operators (beside the upper semicontinuous or compact operators) which has been widely studied in the deterministic case are operators which satisfy Lipschitz-conditions (in the Hausdorff metric); of course all of them are continuous in the sense to be defined below. In this chapter we will prove a result which (roughly spoken) tells us that whenever a (deterministic) fixed point theorem for continuous set-valued operators is valid then the corresponding stochastic fixed point theorem is also valid.

Definition 12. For $A, B \in C B(X)$ let $\rho(A, B)=\sup _{a \in A} d(a, B)$, where $d$ is the distance induced by the norm on $X$, and $D(A, B)=$ $\max \{\rho(A, B), \rho(B, A)\}$. It is well-known that $(C B(X), D)$ is a metric space; $D$ is called "Hausdorff-metric". Let $C: \Omega \rightarrow 2^{X}$ be measurable and $T: \operatorname{Gr} C \rightarrow C B(X)$ a set-valued random operator with stochastic domain $C . \quad T$ is called "continuous" iff for all $\omega \in \Omega, T(\omega, \cdot)$ is continuous as a mapping from $C(\omega)$ into $(C B(X), D)$.

TheOREM 13. Let $C: \Omega \rightarrow 2^{X}$ be separable and $T: \operatorname{Gr} C \rightarrow C B(X)$ a continuous set-valued random operator with stochastic domain $C$ such that for all $\omega \in \Omega,\{x \in C(\omega) / x \in T(\omega, x)\} \neq \varnothing$ ("solvability of the corresponding deterministic fixed point problem"). Then $T$ has a random fixed point.

Proof. We define $N$ : $\operatorname{Gr} C \rightarrow \boldsymbol{R}$ by $N(\omega, x)=d(x, T(\omega, x))$. Let $\omega \in \Omega$ and $x, y \in C(\omega)$ be arbitrary, but fixed. Then

$$
\begin{aligned}
&|N(\omega, x)-N(\omega, y)|=|d(x, T(\omega, x))-d(y, T(\omega, y))| \\
&=\mid D(\{x\}, T(\omega, x))-D(\{x\}, T(\omega, y)) \\
& \quad+D(\{x\}, T(\omega, y))-D(\{y\}, T(\omega, y)) \mid \\
& \leqq|D(T(\omega, x),\{x\})-D(\{x\}, T(\omega, y))|
\end{aligned}
$$




$$
\begin{aligned}
& +|D(\{x\}, T(\omega, y))-D(T(\omega, y),\{y\})| \\
\leqq & D(T(\omega, x), T(\omega, y))+d(x, y) .
\end{aligned}
$$

This shows together with the continuity of $T$ that each $N(\omega, \cdot)$ is continuous on $C(\omega)$.

Now let $x \in X$ and $r \geqq 0$ be chosen arbitrarily, but fixed. Then $\{\omega \in \Omega / x \in C(\omega) \wedge N(\omega, x)<r\}=\{\omega \in \Omega / x \in C(\omega) \wedge T(\omega, x) \cap\{y \in X /\|y-x\|<$ $r\} \neq \phi\} \in \mathscr{A}$. Because of this, the continuity of each $N(\omega, \cdot)$ and the fact, that $\operatorname{Gr} C$ is a measurable subset of $\mathscr{A} \times \mathscr{B}$ ([12, Theorem 3.3]) we have (with $Z$ as a countable set as it appears in Definition 1)

$$
\begin{aligned}
\{(\omega, x) & \in \operatorname{Gr} C / N(\omega, x) \leqq r\} \\
= & \{(\omega, x) \in \Omega \times X / x \in C(\omega) \wedge N(\omega, x) \leqq r\} \\
= & \bigcap_{n \in N} \bigcup_{z \in Z}\left[\left(\left\{\omega \in \Omega / z \in C(\omega) \wedge N(\omega, z)<r+n^{-1}\right\}\right.\right. \\
& \left.\left.\times\left\{x \in X /\|x-z\|<n^{-1}\right\}\right) \cap \operatorname{Gr} C\right] \in \mathscr{A} \times \mathscr{B} .
\end{aligned}
$$

So $N$ is a measurable function from the measurable space $(\mathrm{Gr} C$, $\operatorname{Gr} C \cap(\mathscr{A} \times \mathscr{B}))$ into $R$. Especially $\{(\omega, x) \in \operatorname{Gr} C / N(\omega, x)=0\} \in$ $\mathscr{A} \times \mathscr{B}$.

Now we define $F: \Omega \rightarrow\{A / A \subseteq X\}$ by $F(\omega):=\{x \in X / x \in C(\omega) \cap$ $T(\omega, x)\}$. By assumption each $F(\omega)$ is nonvoid. We choose $\omega \in \Omega$ arbitrary, but fixed. Let $\left(x_{k}\right) \in F(\omega)^{N}$ such that $\left(x_{k}\right) \rightarrow x$. Then $x \in C(\omega)$ and because of

$$
d(x, T(\omega, x)) \leqq\left\|x-x_{k}\right\|+d\left(x_{k}, T\left(\omega, x_{k}\right)\right)+D\left(T\left(\omega, x_{k}\right), T(\omega, x)\right)
$$

and the continuity of $T(\omega, \cdot)$ we have $x \in F(\omega)$. Therefore each $F(\omega)$ is closed and we can proceed analogously to the proof of Theorem 7.

REMARK 14. If we specialize Theorem 13 to the case where $T$ is single-valued, we get a generalization of [6, Theorem 8].

CoROllary 15. Let $C: \Omega \rightarrow 2^{X}$ be separable and $T: \operatorname{Gr} C \rightarrow C B(X)$ a set-valued random operator with stochastic domain $C$ such that for all $\omega \in \Omega$ there exists a $k(\omega) \in[0,1)$ such that for all $x, y \in C(\omega)$, $D(T(\omega, x), T(\omega, y)) \leqq k(\omega) \cdot\|x-y\|$. Furthermore we assume that for all $\omega \in \Omega$ and $x \in b d C(\omega), T(\omega, x) \leqq C(\omega)$. Then $T$ has a random fixed point.

Proof. Because of [1, Theorem 1] Theorem 13 is applicable.

REMARK 16. Corollary 15 generalizes the main result of [13] (in the Banach space case). Itoh's result was formulated in Polish spaces. If we choose our "domain-function" $C$ to be constant, we 
could prove Theorem 13 also in the case where $X$ is merely a Polish space and $T$ defined an $\Omega \times X$. The proof carries over with the obvious changes. So we could also get Itoh's theorem in its original formulation as a corollary to Theorem 13 using [16, Theorem 5].

Definition 17. A Banach space $Y$ is called "Opial space" iff for all sequences $\left(x_{n}\right)$ in $Y$ which converge weakly to $x \in Y$ and all $y \neq x$ we have $\lim \inf \left\|x_{n}-y\right\|>\lim \inf \left\|x_{n}-x\right\|$.

Z. Opial introduced this condition in [17] as a property of Hilbert spaces. For a discussion of Opial spaces see there and in [8], [15].

Corollary 18. Let $X$ be a separable Opial space, $C: \Omega \rightarrow C C(X)$ separable such that for all $\omega \in \Omega, C(\omega)$ is weakly compact and $T$ : $\operatorname{Gr} C \rightarrow C B(X)$ a set-valued random operator with stochastic domain $C$ such that for all $(\omega, x) \in \operatorname{Gr} C, T(\omega, x)$ is compact and for all $\omega \in \Omega$ and $x \in b d C(\omega), T(\omega, x) \leqq C(\omega)$. Furthermore we assume that for all $\omega \in \Omega$ and $x, y \in C(\omega), D(T(\omega, x), T(\omega, y)) \leqq\|x-y\|$. Then $T$ has a random fixed point.

Proof. Because of [1, Theorem 2] Theorem 13 is applicable.

REMARK 19. Throughout this paper separability plays an important role. As far as the separability of the domain function is concerned, this is not too restrictive for applications (cf. the remark after Definition 1). Of course it might be desirable to get random fixed point theorems in the case where $X$ is not separable. But at the present time there seems to be little hope to get such results with methods similiar to those used in this paper. Up to now not even measurability theory for set-valued maps with values in nonseparable spaces seems to be developed. And even for single-valued functions the situation seems to be a little bit pathological: The sum of two measurable (single-valued) functions with values in a nonseparable Banach space need not be measurable. Considering this, one has to ask if one really desires to get random fixed point theorems in nonseparable spaces!

\section{REFERENCES}

1. N. A. Assad and W. A. Kirk, Fixed point theorems for set-valued mappings of contractive type, Pacific J. Math., 43 (1972), 553-562.

2. C. Berge, Espaces topologiques (Fonctions multivoques), Dunod. Paris 1959.

3. A. T. Bharucha-Reid, Fixed point theorems in probabilistic analysis, Bull. of the Amer. Math. Soc., 82, 5 (1976), 641-657.

4. H. F. Bohnenblust and S. Karlin, On a theorem of Ville, in: H. W. Kuhn and A. W. 
Tucker (ed.), Contributions to the theory of games, Princeton University Press, Princeton 1950 .

5. H. W. Engl, Some random fixed point theorems for strict contractions and nonexpansive mappings, Nonlinear Analysis, (to appear).

6. - A general stochastic fixed point theorem for continuous random operators on stochastic domains, J. Math. Anal. Appl., (to appear).

7. - Weak convergence of Mann iteration for nonexpansive mappings without convexity assumptions, Boll. Un. Mat. Ital., (to appear).

8. G. Gossez and E. Lami Dozo, Some geometric properties related to the fixed point theory for nonexpansive mappings, Pacific J. Math., 40, 3 (1972), 565-573.

9. S. Hahn, Fixpunktsätze für mengenwertige Abbildungen in lokalkonvexen Räumen, Math. Nachr., 73 (1976),269-283.

10. O. Hanš, Random fixed point theorems, Trans. 1st Prague Conf. on Information Theory, Statist. Decision Functions, and Random Processes (Liblice, 1956), Czechoslovak Acad. Sci., Prague 1957, 105-125.

11. F. Hausdorff, Mengenlehre, 3. Auflage, de Gruyter, Berlin 1935.

12. C. J. Himmelberg, Measurable relations, Fundamenta Math. LXXXVII (1975), 53-72.

13. S. Itoh, $A$ random fixed point theorem for a multivalued contraction mapping, Pacific J. Math., 68 (1977), 85-90.

14. K. Kuratowski and C. Ryll-Nardzewski, A general theorem on selectors, Bull. Acad. Pol. Sc. (Sér. math., astr. et phys.) XIII (1965), 397-403.

15. E. Lami Dozo, Multivalued nonexpansive mappings and Opial's condition, Proc. of the Amer. Math. Soc., 38, 2 (1973), 286-292.

16. S. B. Nadler, Jr., Multivalued contraction mappings, Pacific J. Math., 30 (1969), 475-488.

17. Z. Opial, Weak convergence of the sequence of successive approximations for nonexpansive mappings, Bull. of the Amer. Math. Soc., 73 (1967), 591-597.

18. D. R. Smart, Fixed Point Theorems, Cambridge University Press, Cambridge 1974.

Received May 25, 1977 and in revised form October 10, 1977. Supported in part by a Auslandsstipendium des österreichischen Bundesministeriums für Wissenschaft und Forschung (Austria).

Georgia Institute of Technology

Atlanta, GA 30332

Permanent Address: Institut für Mathematik Johannes-Kepler-Universität A-4045 Linz, Austria 


\section{PACIFIC JOURNAL OF MATHEMATICS}

\section{EDITORS}

RICHARD ARENS (Managing Editor)

University of California

Los Angeles, CA 90024

Charles W. Curtis

University of Oregon

Eugene, OR 97403

C. C. MOORE

University of California

Berkeley, CA 94720

\section{J. DugundJI}

Department of Mathematics

University of Southern California

Los Angeles, CA 90007

R. FinN and J. Milgram

Stanford University

Stanford, CA 94305

\section{ASSOCIATE EDITORS}
E. F. BECKENBACH
B. H. NeumanN
F. WOLF
K. YosHIDA

\section{SUPPORTING INSTITUTIONS}

\author{
UNIVERSITY OF BRITISH COLUMBIA \\ CALIFORNIA INSTITUTE OF TECHNOLOGY \\ UNIVERSITY OF CALIFORNIA \\ MONTANA STATE UNIVERSITY \\ UNIVERSITY OF NEVADA, RENO \\ NEW MEXICO STATE UNIVERSITY \\ OREGON STATE UNIVERSITY \\ UNIVERSITY OF OREGON
}

\author{
UNIVERSITY OF SOUTHERN CALIFORNIA \\ STANFORD UNIVERSITY \\ UNIVERSITY OF HAWAII \\ UNIVERSITY OF TOKYO \\ UNIVERSITY OF UTAH \\ WASHINGTON STATE UNIVERSITY \\ UNIVERSITY OF WASHINGTON
}

The Supporting Institutions listed above contribute to the cost of publication of this Journal, but they are not owners or publishers and have no responsibility for its content or policies.

Mathematical papers intended for publication in the Pacific Journal of Mathematics should be in typed form or offset-reproduced, (not dittoed), double spaced with large margins. Please do not use built up fractions in the text of the manuscript. However, you may use them in the displayed equations. Underline Greek letters in red, German in green, and script in blue. The first paragraph or two must be capable of being used separately as a synopsis of the entire paper. Items of the bibliography should not be cited there unless absolutely necessary, in which case they must be identified by author and journal, rather than by item number. Manuscripts, in triplicate, may be sent to any one of the editors. Please classify according to the scheme of Math. Reviews, Index to Vol. 39. All other communications should be addressed to the managing editor, or Elaine Barth, University of California, Los Angeles, California, 90024.

50 reprints to each author are provided free for each article, only if page charges have been substantially paid. Additional copies may be obtained at cost in multiples of 50 .

The Pacific Journal of Mathematics is issued monthly as of January 1966. Regular subscription rate: $\$ 72.00$ a year (6 Vols., 12 issues). Special rate: $\$ 36.00$ a year to individual members of supporting institutions.

Subscriptions, orders for numbers issued in the last three calendar years, and changes of address should be sent to Pacific Journal of Mathematics, 103 Highland Boulevard, Berkeley, California, 94708. Older back numbers obtainable from Kraus Periodicals Co., Route 100, Millwood, NY 10546.

PUBLISHED BY PACIFIC JOURNAL OF MATHEMATICS, A NON-PROFIT CORPORATION

Printed at Kokusai Bunken Insatsusha (International Academic Printing Co., Ltd.). 8-8, 3-chome, Takadanobaba, Shinjuku-ku, Tokyo 160, Japan.

Copyright (C) 1978 by Pacific Journal of Mathematics Manufactured and first issued in Japan 


\section{Pacific Journal of Mathematics}

\section{Vol. 76, No. $2 \quad$ December, 1978}

Stephanie Brewster Brewer Taylor Alexander, Local and global convexity in complete Riemannian manifolds ...........................

Claudi Alsina i Català, On countable products and algebraic convexifications of probabilistic metric spaces ...............................

Joel David Berman and George Grätzer, Uniform representations of

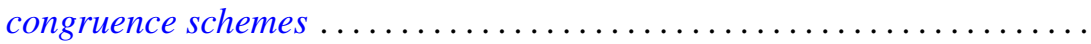

Ajit Kaur Chilana and Kenneth Allen Ross, Spectral synthesis in

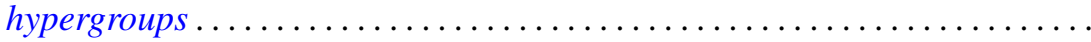

David Mordecai Cohen and Howard Leonard Resnikoff, Hermitian quadratic forms and Hermitian modular forms . .........................

Frank Rimi DeMeyer, Metabelian groups with an irreducible projective

representation of large degree .............................

Robert Ellis, The Furstenberg structure theorem .....................

Heinz W. Engl, Random fixed point theorems for multivalued mappings .......

William Andrew Ettling, On arc length sharpenings ..................

Kent Ralph Fuller and Joel K. Haack, Rings with quivers that are trees........

Kenneth R. Goodearl, Centers of regular self-injective rings ...............

John Gregory, Numerical algorithms for oscillation vectors of second order

differential equations including the Euler-Lagrange equation for

symmetric tridiagonal matrices.

Branko Grünbaum and Geoffrey Shephard, Isotoxal tilings

Myron Stanley Henry and Kenneth Leroy Wiggins, Applications of

approximation theory to differential equations with deviating

arguments

Mark Jungerman, The non-orientable genus of the n-cube .

Robert Richard Kallman, Only trivial Borel measures on $S_{\infty}$ are

quasi-invariant under automorphisms ................

Joyce Longman and Michael Rich, Scalar dependent algebras in the

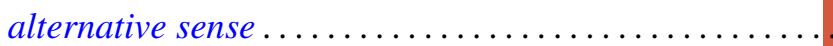

Richard A. Mollin, The Schur group of a field of characteristic zero ........ 471

David Pokrass, Some radical properties of rings with $(a, b, c)=(c, a, b) \ldots 479$

Margaret Shay and Paul Ruel Young, Characterizing the orders changed by

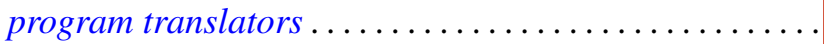

Jerrold Norman Siegel, On the structure of $B_{\infty}(F), F$ a stable space...

Surjeet Singh, (hnp)-rings over which every module admits a basic

submodule...

A. K. Snyder, Universal interpolating sets and the Nevanlinna-Pick property in

Banach spaces of functions...

Jeffrey D. Vaaler, On the metric theory of Diophantine approximation ... 